\title{
Binding Energies and Pairing Gaps in Semi-Magic Nuclei Obtained Using New Regularized Higher-Order EDF Generators
}

\author{
Bennaceur, K. \\ WORLD SCIENTIFIC PUBL CO PTE LTD \\ 2017
}

Bennaceur, K , Dobaczewski , J \& Gao , Y 2017 , Binding Energies and Pairing Gaps in Semi-Magic Nuclei Obtained Using New Regularized Higher-Order EDF Generators . in J H Hamilton , A V Ramayya \& P Talou (eds), Fission And Properties Of Neutron-rich Nuclei : Proceedings of the Sixth International Conference on Icfn6. WORLD SCIENTIFIC PUBL CO PTE LTD , pp. 27-34 , 6th International Conference on Fission and Properties of Neutron-Rich Nuclei (ICFN) , 06/11/2016 . https://doi.org/10.1142/9789813229426_0007

http://hdl.handle.net/10138/307665

https://doi.org/10.1142/9789813229426_0007

other

acceptedVersion

Downloaded from Helda, University of Helsinki institutional repository.

This is an electronic reprint of the original article.

This reprint may differ from the original in pagination and typographic detail.

Please cite the original version. 


\title{
Binding energies and pairing gaps in semi-magic nuclei obtained using new regularized higher-order EDF generators
}

\author{
K. Bennaceur, ${ }^{1,2,3}$ J. Dobaczewski, ${ }^{2,3,4,5}$ and Y. Gao (高原) ${ }^{6}$ \\ ${ }^{1}$ Univ Lyon, Université Lyon 1, CNRS/IN2P3, IPNL, F-69622 Villeurbanne, France \\ ${ }^{2}$ Department of Physics, PO Box 35 (YFL), FI-40014 University of Jyväskylä, Finland \\ ${ }^{3}$ Helsinki Institute of Physics, P.O. Box 64, FI-00014 University of Helsinki, Finland \\ ${ }^{4}$ Department of Physics, University of York, Heslington, York YO10 5DD, United \\ Kingdom \\ ${ }^{5}$ Institute of Theoretical Physics, Faculty of Physics, University of Warsaw, ul. \\ Pasteura 5, PL-02-093 Warsaw, Poland \\ ${ }^{6}$ State Key Laboratory of Nuclear Physics and Technology, School of Physics, Peking \\ University, Beijing 100871, China
}

\begin{abstract}
We present results of the Hartree-Fock-Bogolyubov calculations performed using nuclear energy density functionals based on regularized functional generators at next-to-leading and next-to-next-to-leading order. We discuss properties of binding energies and pairing gaps determined in semi-magic spherical nuclei. The results are compared with benchmark calculations performed for the functional generator SLyMR0 and functional UNEDF0.

Keywords: nuclear energy density functionals, regularized functional generators, nuclear binding energies, pairing gaps
\end{abstract}

\section{Introduction}

A quest for energy density functionals (EDFs) that would precisely and accurately describe multitude of low-energy nuclear properties is at present one of the most important research avenues in nuclear physics. The problem has been addressed in quite a number of recent studies, ${ }^{1-10}$ where various extensions of the standard EDFs, used for the last 60-odd years, were proposed.

In this conference communication, we present results of calculations performed for semi-magic nuclei across the mass chart, using the newly developed EDFs based on the regularized higher-order generators with pairing. ${ }^{10}$ As discussed in Ref. 10, the proposed new parametrizations at nextto-leading order (NLO) REG2c.161026 and next-to-next-to-leading $\left(\mathrm{N}^{2} \mathrm{LO}\right)$ REG4c.161026 correspond to a fairly low effective mass and overestimate pairing strength. Therefore, they are not good enough to embark for them 
on massive mass-table calculations. However, inexpensive calculations performed for spherical semi-magic nuclei, which are reported on in this paper, can constitute a useful illustration of the overall bulk properties corresponding to the newly developed EDFs.

\section{Results}

We performed the Hartree-Fock-Bogolyubov (HFB) calculations for all bound semi-magic nuclei across the mass chart, using the NLO REG2c.161026 and $\mathrm{N}^{2} \mathrm{LO}$ REG4c.161026 EDFs, ${ }^{10}$ ) SLyMR0, ${ }^{7}$ ) and UNEDF0. ${ }^{11}$ ) For UNEDF0, the Lipkin-Nogami (LN) method was used to account for approximate particle-number restoration as in Ref. 12. For the finite-range NLO and $\mathrm{N}^{2} \mathrm{LO}$ generators, we solved the non-local selfconsistent equations using the newly developed code FINRES $_{4}$ (Finite-Range Self-consistent Spherical Space-coordinate Solver), ${ }^{13}$ which is based on the method proposed by Hooverman. ${ }^{14}$ For the zero-range generator SLyMR0, we obtained the solutions using the spherical solver LENTEUR, ${ }^{15}$ and for the quasi-local functional UNEDF0, using the code HOSPHE, ${ }^{16,17}$ similarly as in Ref. 18.

For NLO, $\mathrm{N}^{2} \mathrm{LO}$, and UNEDF0, where the fit covariance matrices are known, we determined statistical uncertainties of all calculated observables according to the methodology presented in Ref. 20. For SLyMR0, only the values of observables were determined.

In Figs. 1 and 2, for nuclei where the binding energies are known from experiment or systematics, ${ }^{19}$ ) we show the binding-energy residuals determined for the four studied EDFs. We note that for SLyMR0, the obtained differences between theory and experiment are significantly larger than those obtained for the other three EDFs, and therefore, in the figures they were divided by a factor of five. We also note that NLO, $\mathrm{N}^{2} \mathrm{LO}$, and SLyMR0 EDFs are built as exact averages of two-body or many-body generators, both in the particle-hole and pairing channels, and therefore, they are suitable without ambiguity for beyond-mean-field and symmetryrestoration calculations. On the other hand, EDF UNEDF0 is built as an average of a density-dependent two-body Skyrme-type generators that are different in particle-hole and pairing channels and with some omitted terms in the particle-hole channel.

Although the pattern of comparison with data is fairly different among all four studied EDFs, we clearly see that the new NLO and $\mathrm{N}^{2} \mathrm{LO}$ EDFs describe data better than SLyMR0 (recall the scaling factor of five used 

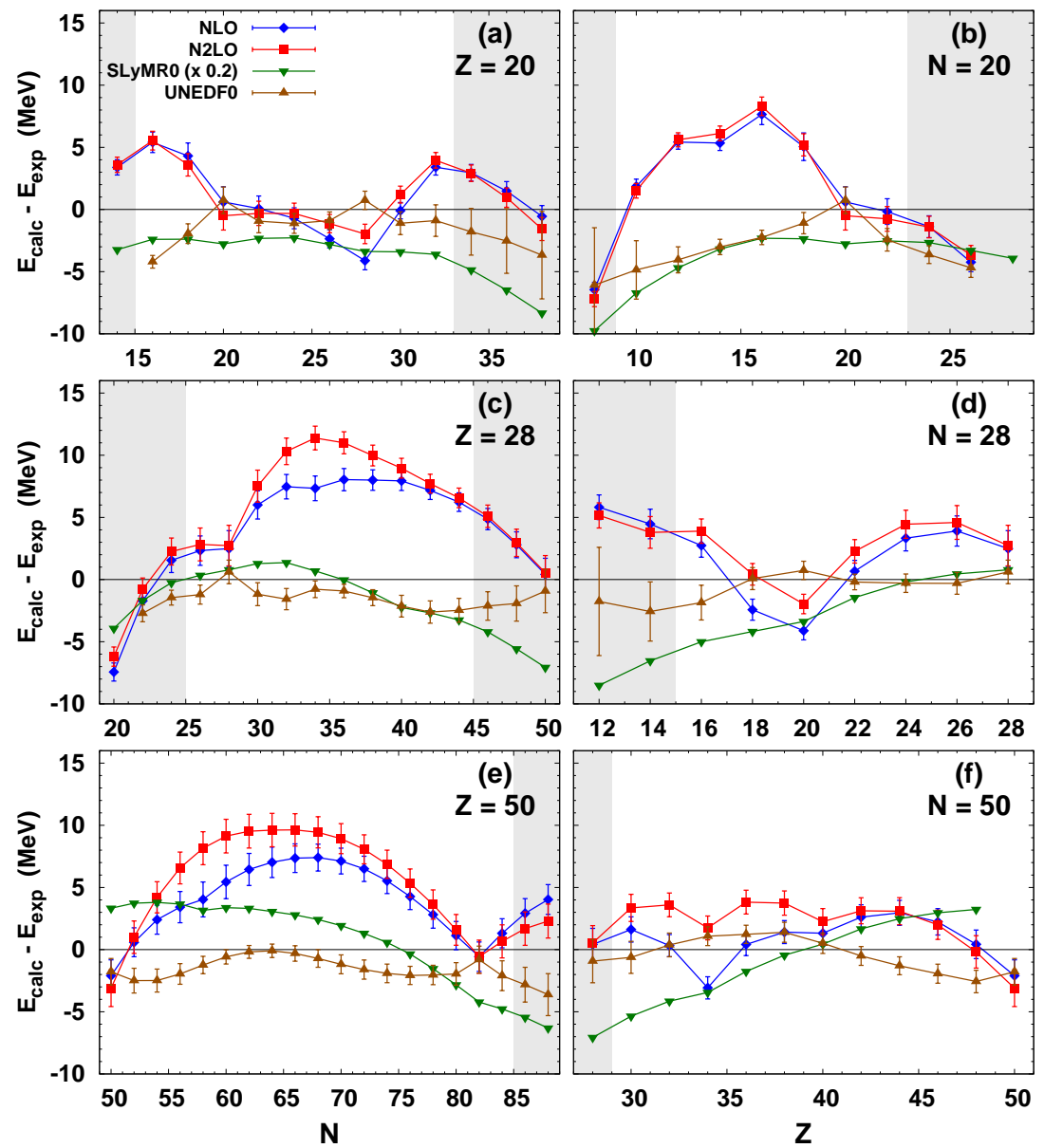

Fig. 1. Differences between calculated (HFB results) and experimental (AME12 ${ }^{19}$ ) ground-state energies of proton-magic (left panels) and neutron-magic nuclei with $Z, N=20,28$, or 50. Calculations were performed using EDFs NLO REG2c.161026 ${ }^{10}$ ) (diamonds), $\mathrm{N}^{2} \mathrm{LO}$ REG4c.161026 ${ }^{10}$ ) (squares), SLyMR0 ${ }^{7}$ ) (down triangles), and UNEDF0 ${ }^{11}$ ) (up triangles). For SLyMR0, to fit in the figure, the differences were divided by a factor of five. Shaded zones correspond to the AME12 masses taken from systematics.

for SLyMR0). However, results obtained at NLO and $\mathrm{N}^{2} \mathrm{LO}$ are still fairly worse than those obtained for the standard Skyrme-like EDF UNEDF0. In particular, for the NLO and $\mathrm{N}^{2} \mathrm{LO}$ EDFs, we see conspicuous "arches" of residuals between the doubly magic nuclei. Usually these feature of calcu- 


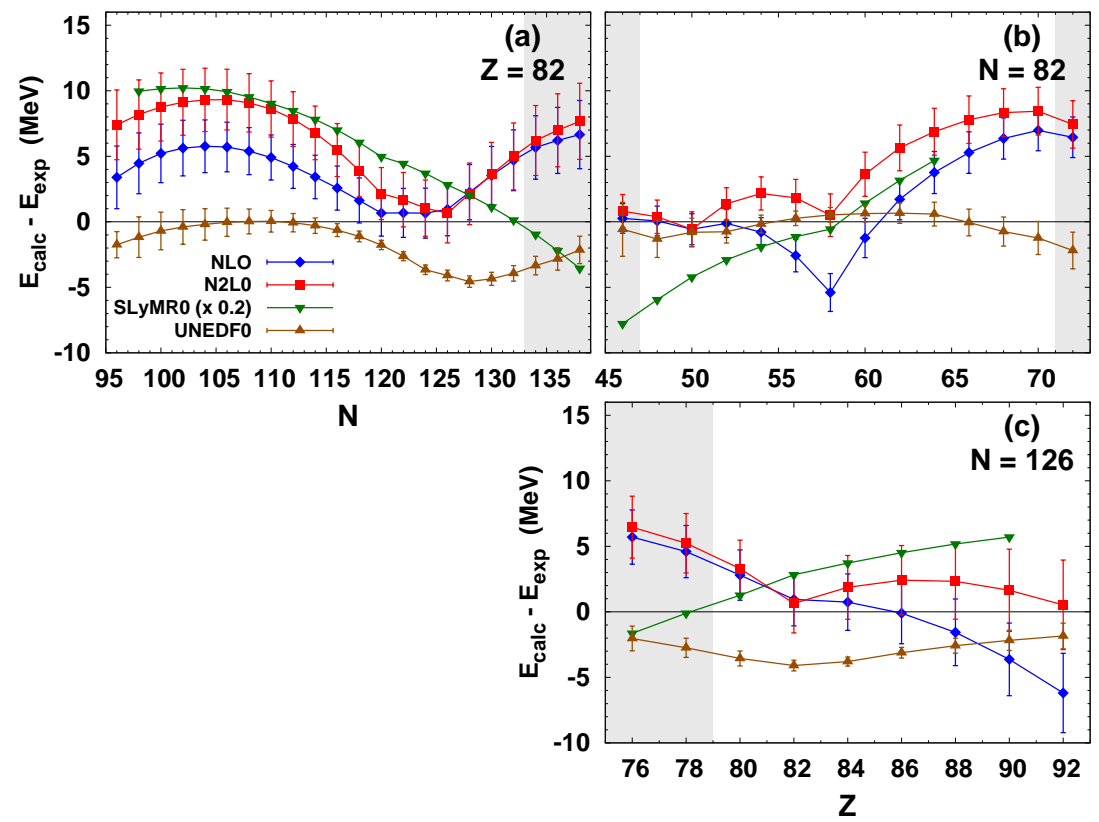

Fig. 2. Same as in Fig. 1 but for the semi-magic nuclei with $Z, N=82$ and $N=126$.

lated ground-state energies was attributed to low effective mass, however, we note here that for SLyMR0, which has a low effective mass too, no such an effect is seen. We also note that the statistical uncertainties obtained for UNEDF0 significantly increase when going towards neutron-rich nuclei, ${ }^{18}$ whereas those for the NLO and $\mathrm{N}^{2} \mathrm{LO}$ EDFs depend on the neutron excess much less.

In Figs. 3 and 4, we show neutron and proton pairing gaps calculated as pairing fields averaged with density matrices, ${ }^{12}$ and for the LN method (UNEDF0), corrected by adding the corresponding $\lambda_{2}$ LN parameters. ${ }^{12}$ As discussed in Ref. 10, for the NLO and $\mathrm{N}^{2} \mathrm{LO}$ EDFs, pairing correlations were adjusted to values largely overestimating experimental data. This feature is clearly visible in the figures, and should certainly be improved upon in future planned adjustment of parameters. We also see that the HFB results shown for the NLO, $\mathrm{N}^{2} \mathrm{LO}$, and SLyMR0 EDFs exhibit unphysical breaking of pairing at doubly magic gaps, which is a feature related to the lack of particle-number restoration, and thus is absent in the HFB+LN results shown for UNEDF0. 

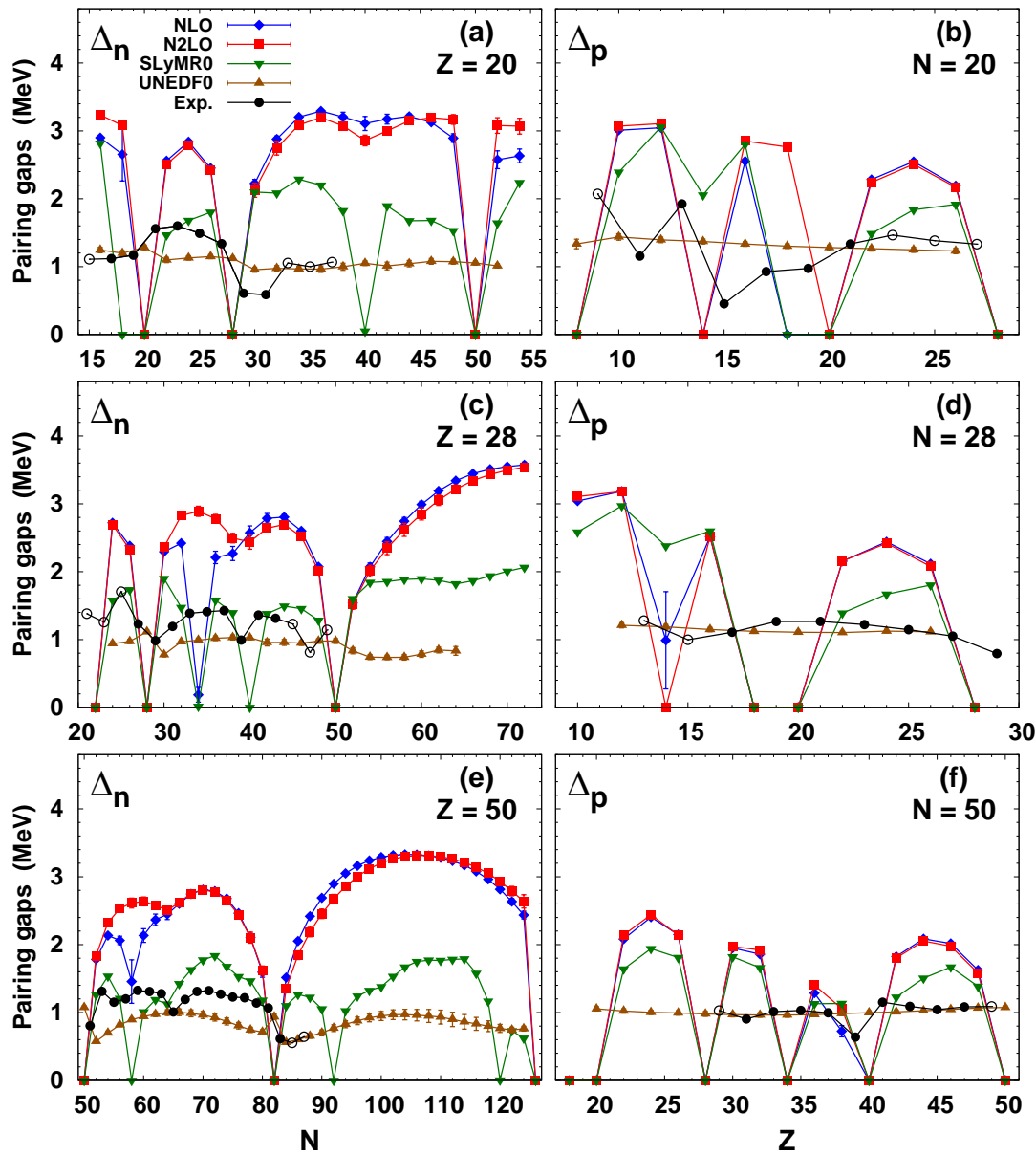

Fig. 3. Calculated (HFB results) and experimental (AME12 ${ }^{19}$ ) neutron pairing gaps of proton-magic nuclei (left panels) and proton pairing gaps of neutron-magic nuclei with $Z, N=20,28$, or 50 . Calculations were performed using EDFs NLO REG2c.161026 ${ }^{10}$ ) (diamonds), $\mathrm{N}^{2} \mathrm{LO}$ REG4c.161026 ${ }^{10}$ ) (squares), SLyMR0 ${ }^{7}$ ) (down triangles), and UNEDF0 ${ }^{11}$ ) (up triangles). Experimental values (circles) correspond to the three-point mass staggering centered at odd particle numbers. ${ }^{21}$ ) Open circles indicate values, for which at least one of the three AME12 masses were taken from systematics.

\section{Conclusions}

In this article we presented the binding-energy residuals and average pairing gaps obtained for semi-magic nuclei using two recently adjusted finite-range 


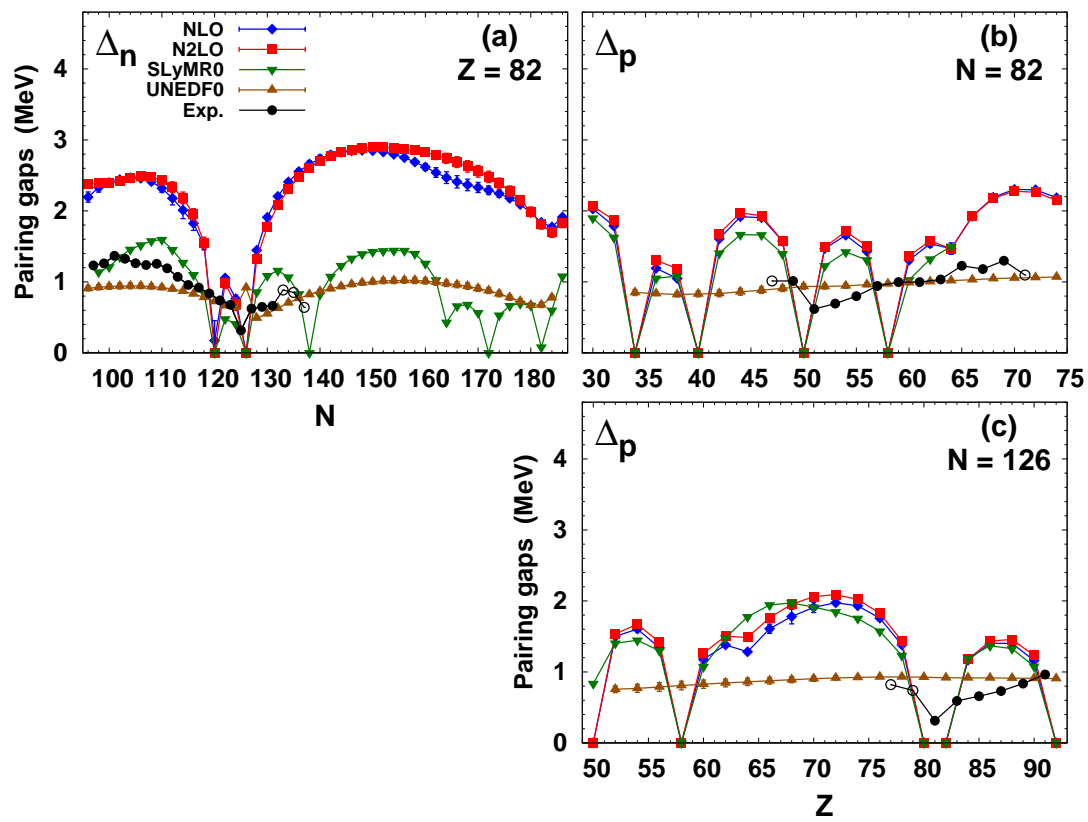

Fig. 4. Same as in Fig. 3 but for the semi-magic nuclei with $Z, N=82$ and $N=126$.

pseudopotentials at NLO and $\mathrm{N}^{2} \mathrm{LO}$, as well as the EDF UNEDF0 and zerorange pseudopotential SLyMR0. For all cases but SLyMR0, the propagated statistical errors of observables were calculated.

For the set of nuclei considered here, and for finite-range pseudopotentials, the average deviations between experimental and calculated binding energies are larger than those obtained for EDF UNEDF0, but they are significantly smaller than the ones obtained for the zero-range pseudopotential SLyMR0. For the NLO and $\mathrm{N}^{2} \mathrm{LO}$ EDFs, the typical arches that appear in the binding-energy residuals between major shells might be due to their very low effective masses (close to 0.4). However, the fact that similar arches do not appear for SLyMR0, which has an effective mass of 0.47 , questions this conjecture.

Based on the results obtained for binding-energy residuals and average pairing gaps, there was no clear improvement when going from the finiterange pseudopotential at $\mathrm{NLO}$ to the one at $\mathrm{N}^{2} \mathrm{LO}$. This does not necessarily mean that the additional degrees of freedom introduced at $\mathrm{N}^{2} \mathrm{LO}$ are not relevant, but most likely reflects the fact that the penalty function based 
on spherical doubly magic nuclei did not allow for properly constraining them.

The next step in the development of this family of pseudopotentials will consist in increasing the effective mass. The obvious way to do this is to introduce three-body terms in the pseudopotential. The present computational ressources restrict this extension to the introduction of zero-range three-body terms in the spirit of the work of Onishi and Negele ${ }^{22}$ and will require the use of a cut-off to prevent the divergence of the energy. The work along these lines is in progress.

\section{Acknowledgments}

This work was supported by the Academy of Finland, the University of Jyväskylä within the FIDIPRO program, by the CNRS/IN2P3 through PICS No. 6949.

\section{References}

1. B. G. Carlsson, J. Dobaczewski and M. Kortelainen, Local nuclear energy density functional at next-to-next-to-next-to-leading order, Phys. Rev. C 78, p. 044326 (2008).

2. M. Zalewski, J. Dobaczewski, W. Satuła and T. R. Werner, Spinorbit and tensor mean-field effects on spin-orbit splitting including selfconsistent core polarizations, Phys. Rev. C 77, p. 024316 (Feb 2008).

3. F. Raimondi, B. G. Carlsson and J. Dobaczewski, Effective pseudopotential for energy density functionals with higher-order derivatives, Phys. Rev. C 83, p. 054311 (2011).

4. J. Dobaczewski, K. Bennaceur and F. Raimondi, Effective theory for low-energy nuclear energy density functionals, J. Phys. G 39, p. 125103 (2012).

5. F. Raimondi, K. Bennaceur and J. Dobaczewski, Nonlocal energy density functionals for low-energy nuclear structure, J. Phys. G: Nucl. Part. Phys. 41, p. 055112 (2014).

6. K. Bennaceur, J. Dobaczewski and F. Raimondi, Extended skyrme pseudopotential deduced from infinite nuclear matter properties, EPJ Web of Conf. 66, p. 02031 (2014).

7. J. Sadoudi, M. Bender, K. Bennaceur, D. Davesne, R. Jodon and T. Duguet, Skyrme pseudo-potential-based EDF parametrization for spuriosity-free MR EDF calculations, Phys. Scr. T154, p. 014013 (2013). 
8. J. Sadoudi, T. Duguet, J. Meyer and M. Bender, Skyrme functional from a three-body pseudopotential of second order in gradients: Formalism for central terms, Phys. Rev. C 88, p. 064326 (2013).

9. D. Davesne, J. Navarro, P. Becker, R. Jodon, J. Meyer and A. Pastore, Extended skyrme pseudopotential deduced from infinite nuclear matter properties, Phys. Rev. C 91, p. 064303 (Jun 2015).

10. K. Bennaceur, A. Idini, J. Dobaczewski, P. Dobaczewski, M. Kortelainen and F. Raimondi, arXiv:1611.09311.

11. M. Kortelainen, T. Lesinski, J. Moré, W. Nazarewicz, J. Sarich, N. Schunck, M. V. Stoitsov and S. Wild, Nuclear energy density optimization, Phys. Rev. C 82, p. 024313 (Aug 2010).

12. M. V. Stoitsov, J. Dobaczewski, W. Nazarewicz, S. Pittel and D. J. Dean, Systematic study of deformed nuclei at the drip lines and beyond, Phys. Rev. C 68, p. 054312 (2003).

13. Bennaceur K. et al. 2017, to be submitted to Computer Physics Communications.

14. R. H. Hooverman, A technique for numerical solution of the Schroedinger equation with non-local potentials, Nuclear Physics A 189, 155 (1972).

15. K. Bennaceur, LENTEUR HFB code unpublished.

16. B. Carlsson, J. Dobaczewski, J. Toivanen and P. Veselý, Solution of selfconsistent equations for the N3LO nuclear energy density functional in spherical symmetry. The program Hosphe (v1.02), Comput. Phys. Comm. 181, p. 1641 (2010).

17. B. G. Carlsson, J. Toivanen, P. Veselý, and Y. Gao, to be published.

18. Y. Gao, J. Dobaczewski, M. Kortelainen, J. Toivanen and D. Tarpanov, Propagation of uncertainties in the skyrme energy-density-functional model, Phys. Rev. C 87, p. 034324 (2013).

19. M. Wang, G. Audi, A. H. Wapstra, F. G. Kondev, M. MacCormick, X. Xu and B. Pfeiffer, The AME2012 atomic mass evaluation (ii). tables, graphs and references, Chin. Phys. C 36, 1603 (2012).

20. J. Dobaczewski, W. Nazarewicz and P.-G. Reinhard, Error Estimates of Theoretical Models: a Guide, J. Phys. G: Nucl. Part. Phys. 41, p. 074001 (2014).

21. W. Satuła, J. Dobaczewski and W. Nazarewicz, Odd-even staggering of nuclear masses: Pairing or shape effect?, Phys. Rev. Lett. 81, 3599 (1998).

22. N. Onishi and J. Negele, Two-body and three-body effective interactions in nuclei, Nuclear Physics A 301, 336 (1978). 